\title{
Editorial
}

\section{Physical Activity and HIV in Africa}

\author{
Clemens Ley ${ }^{*}, 1$, and António Prista ${ }^{2, \S}$
}

\author{
${ }^{1}$ Institut für Sportwissenschaft, Universität Wien, Austria \\ ${ }^{2}$ Centro de Investigação e Desenvolvimento do Desporto e Actividade Física, Universidade Pedagógica de \\ Moçambique, Mozambique
}

Over the past decades evidence has emerged regarding the benefits of exercise on physical health and quality of life of people living with HIV [1-4]. Regular exercise training is considered safe for people living with HIV and important complementary means to address effectively health problems and side effects of medication. Therefore, it is strongly recommended that people living with HIV augment their physical activity levels, adequate information and awareness is spread among health workers and trainers, and corresponding opportunities for physical activity are built and maintained.

However, many questions still remain unanswered, such as how to achieve these positive effects in the diverse and specific living contexts of the people, and particularly in Africa, where the main burden of HIV is found, but where few studies have been conducted. "Conclusions drawn from women [with HIV] living in a Western society will not necessarily reflect those found in sub-Saharan Africa where socio-economic factors and ethnicity could influence body composition changes" $[5,6]$.

In that regard, the international conference on Physical Activity and Sports for Health and Development in Africa (PASHDA) was celebrated in March 2014 in order to discuss particularly the situation of physical activity and health in Africa, but also what other countries can learn from Africa and vice versa. The conference was attended by 223 delegates coming from 14 countries and covering topics of Physical Activity in Africa related to Health, Urban Planning, Sedentary, Aging and Management of NonCommunicable diseases (NCDs), as well as Body Culture and Games in the African Traditional Societies. A specific symposium on Physical Activity and HIV was conducted during the conference to dialogue about the state-of-the-art and the role of physical activity in regards to HIV. Research from African countries and Brazil was discussed from different angles and disciplines obtaining a holistic view on this complex topic. Some of the results from the symposium are presented in this thematic issue:

\footnotetext{
*Address correspondence to this author at the Institut für Sportwissenschaft, University of Vienna, Auf der Schmelz 6a, 1150 Wien, Austria; Tel: 0043 6991720 8761; E-mail: clemensley@gmail.com

${ }^{\S}$ Guest Editor
}

The first article raises the issue of cardiac diseases in people living with HIV. While the life expectancy of people with HIV is increasing, the relationship between HIV infection and risks for cardiac diseases is gaining weight. This is also true in some countries in Southern Africa where obesity is becoming an increasing problem [7]. The introductory research letter from Dr. Mocumbi (Mozambique) is followed by a systematic review, conducted by Dr. Leach and colleagues (South Africa), focusing on the effects of exercise on body composition in people living with HIV The review discusses the existing evidence and calls for further research on this particularly topic for people living with HIV. Three original research articles respond to this call, presenting data from three different studies in Mozambique, South Africa and Brazil. The first discusses effects of a supervised exercise program on body composition and muscular performance, which was conducted throughout two years in the laboratory of Physical Activity and Health Promotion at the Rio de Janeiro State University. This program included aerobic, strength and flexibility exercises; meanwhile the research conducted at the Pedagogic University Mozambique compared different types of exercise, including also active games. Playful exercising seems as effective as regular exercising and might therefore be used as an alternative. Comparing different types of physical activity aims to optimise the choice of exercise and the implementation of adequate programs in different contexts. The last article addresses challenges for practising sport and exercise in the context of HIV, especially referring to HIV-related stigma and motives. It explores social-ecological, motivational and volitional factors for initiating and maintaining physical activity among people living with HIV and concludes practical recommendations.

In total, the symposium showed the importance to maintain a holistic work and research approach. It concluded that the actual state-of-the-art must be increased through the conduction of holistic and multiples studies. On the one hand, there is a need to increase the understanding of the effects of exercise on physical and psychosocial health of people living with HIV, particularly in Africa and in relationship with non-communicable diseases and risk factors. Networking and coordinated research in various countries are envisaged. On the other hand, further knowledge is required to understand how these effects are 
most appropriately achieved in the specific living contexts. Therefore, further research should investigate the effects of different types of physical activity, particularly those types of physical activity that are more likely to be implemented and maintained in the particular setting. It seems critical to obtain a best fit between possible effects and real opportunities to achieve the recommendations, considering social-ecological influences, barriers and motivation of people living with HIV. This is crucial to increment their physical activity levels, to optimise their participation in sport and exercise, and to achieve and maintain on long term the positive effects.

\section{CONFLICT OF INTEREST}

The authors confirm that this article content has no conflict of interest.

\section{ACKNOWLEDGEMENTS}

The symposium was partly funded by the Cooperation for Development Group DIM of the Technical University of Madrid, Spain.

\section{REFERENCES}

[1] Gomes NM, Conceicao CS, Carvalho VO, Brites C. A systematic review of the effects of different types of therapeutic exercise on physiologic and functional measurements in patients with HIV/AIDS. Clinics 2013; 68: 1157-67.

[2] Gomes Neto M, Ogalha C, Andrade AM, Brites C. A systematic review of effects of concurrent strength and endurance training on the health-related quality of life and cardiopulmonary status in patients with HIV/AIDS. Biomed Res Int 2013; 2013: 319524.

[3] O'Brien K, Nixon S, Tynan A-M, Glazier R. Aerobic exercise interventions for adults living with HIV/AIDS. Cochrane Database Syst Rev 2010; 8.

[4] O'Brien K, Tynan A-M, Nixon S, Glazier RH. Effects of progressive resistive exercise in adults living with HIV/AIDS: systematic review and meta-analysis of randomized trials. AIDS Care 2008; 20: 631-53.

[5] Myburgh KH, De Bruto PC. Body composition in women with HIV/AIDS: The relevance of exercise. CME 2008; 26: 339-45.

[6] Chopra M, Ford N. Scaling up health promotion interventions in the era of HIV/AIDS: challenges for a rights based approach. Health Promot Int 2005; 20:383-90.

[7] Lambert EV, Kolbe-Alexander T. Physical Activity and Chronic Diseases of Lifestyle in South Africa. In: Steyn K, Fourié J, Temple N, Eds. Chronic diseases of lifestyle in South Africa: 1995 - 2005. Tygerberg: Medical Research Council 2006; pp. 23-32.

(C) Ley and Prista; Licensee Bentham Open.

This is an open access article licensed under the terms of the Creative Commons Attribution Non-Commercial License (http://creativecommons.org/licenses/by-nc/

3.0/) which permits unrestricted, non-commercial use, distribution and reproduction in any medium, provided the work is properly cited. 